\title{
Evaluation of Drought Tolerant Rice Genotypes in Different Moisture Regime on Western Nepal
}

\section{Santosh Raj Tripath ${ }^{1 *}$, Bhim Nath Adhikari ${ }^{2}$, Keshav Raj Pokhrel ${ }^{3}$ and Susma Adhikari ${ }^{4}$}

${ }^{1}$ Senior Scientist, Regional Agriculture Research Station, Tarahara, Nepal

${ }^{2}$ Senior Scientist, Ginger Research Program, Kapurkot, Nepal

${ }^{3}$ Technical Officer, Agriculture Research Station, Surkhet, Nepal

${ }^{4}$ Technician, Regional Agriculture Research Station, Khajura, Nepal

*Corresponding Author: Santosh Raj Tripath, Senior Scientist, Regional Agriculture Research Station, Tarahara, Nepal.

Received: September 26, 2019; Published: October 22, 2019

DOI: 10.31080/ASAG.2019.03.0692

\begin{abstract}
Field experiments were conducted under irrigated, rainfed and reproductive water stress environment for three successive years (2015-2017) at Regional Agriculture Research Station, Khajura; Regional Agriculture Research Station, Doti and Agriculture Research Station, Surkhet to identify and select high yielding and major diseases and pests' resistant genotypes. Twenty-four genotypes identified from IRRI/STRASA experiment were taken for evaluation in RCB design with 3 replications under low fertilizer dose. Genotypes IR 83376-B-B-7-1, IR 877761-52-1-2-2, IR 95840-33-3-2-1 and IR 82608-B-B-33-2 were found drought resistance, early maturing. So, these genotypes would help to food security in western region of Nepal where average rainfall is low and drought is prevailing during rice growing months.
\end{abstract}

Keywords: Drought; Rice; Rainfed; Genotypes

\section{Introduction}

Rice (Oryza sativa) is most susceptible to damage from water deficit [1]. Drought is a world-wide problem that seriously influences amount and stability of grain production. The effect of drought varies with the variety, degree and duration of stress and its coincidence with different growth stages [2]. The full yield potential of rice on any site in any season is probably never realized. Periods of unsuitable weather causes stress hence reduce the yield [3]. Particularly, drought stress at reproductive stage of the crop has large effect on grain yield under rainfed condition [4]. The percentage of drought affected land area in the World more than doubled from the 1970s to the early 2000s [5]. Increasing human population and global climate change make the situation more serious [6]. It is estimated that $50 \%$ of world rice production is affected by drought [7]. Water deficit is becoming increasingly frequent in irrigated areas due to falling water tables. The interna- tional scarcity of water threatens the sustainability of the irrigated rice ecosystem [8].

In Nepal, rice is grown in about1.5 million ha with total production of 5.60 million tons [9]. Rice area and production in eastern region are higher and decline when move from east to west due to a large variation in rainfall amount, on set of rainfall, varieties grown and differences on socio-economic conditions among the regions. The productivity also shows the same trend from east to west [10]. Rice production and annual rainfall data shows positive response of rice yield with amount of rainfall [11]. Rainy season starts in June15 and end in last of October and during this period, total rainfall ranges from 1200 to $1800 \mathrm{~mm}$ with monthly average $200 \mathrm{~mm}$. Moreover, rice is being affected at different spell of drought severities due to uneven distribution of rainfall during the rice growing season. Rice area was reduced by $13 \%$ mainly in rainfed districts of Nepal due to severe drought incidence in 2006 [12]. 
Rice is grown over a wide range of agro-ecological conditions; from the lowlands in the Terai (70 masl) across high mountain valleys and mountain slopes (3050 masl) in Jumla, the highest ricegrowing location in the world. Therefore, varieties recommended for one region, may not work for all over the country. Thus, this study was conducted to select of drought tolerant rice genotypes under reproductive water stress condition for mid and far western regions.

\section{Materials and Methods}

Location

Field experiments were conducted at Regional Agriculture Research Station (RARS), Khajura, Regional Agriculture Research Station (RARS), Doti and Agriculture Research Station (ARS), Surkhet during rainy seasons of 2015 to 2017. RARS, Khajura, RARS, Doti and ARS, Surkhet lie at the latitudes of $28^{\circ} 06^{\prime} \mathrm{N}, 29^{\circ} 15^{\prime} \mathrm{N}$ and $28^{\circ} 30^{\prime}$ and longitude of $81^{\circ} 37^{\prime} \mathrm{E}, 80^{\circ} 55^{\prime}$ Eand $81047^{\prime}$ with an altitude of $181 \mathrm{~m}, 610 \mathrm{~m}$ and580 meters above from sea level, respectively.

\section{Treatment}

A total twenty-four genotypes in Advanced Yield Trial 100 to 120 days maturity were tested in RCBD. Irrigated and reproductive water stress trials were conducted at RARS Khajura, and rainfed trails were conducted at ARS, Surkhet and RARS, Doti.

\section{Culture practices}

Twenty-five days old seedling was transplanted with two to three seedlings per hill with $20 \mathrm{~cm}$ spacing between hills and between rows. Irrigation was applied at transplanting to until milking for irrigated condition. Drought stress during reproductive stage is created by delaying seed sowing and transplanting by four weeks. The water from experiments was drained off 4 weeks (28 days) after transplanting to induce Reproductive Stress. Fertilizer applied @ 60:30:20 kg/ha of $\mathrm{N}: \mathrm{P}_{2} \mathrm{O}_{5}: \mathrm{K}_{2} \mathrm{O}$. Nitrogen was applied as urea $(46 \% \mathrm{~N})$ on two occasions $(1 / 2$ each at transplanting as a basal and at 30 days after transplanting), while the $\mathrm{P}_{2} \mathrm{O}_{5}$ and $\mathrm{K}_{2} \mathrm{O}$ were applied once as a basal application in form of Di- ammonium phosphate (DAP) (46\% P and 18\% N) and of Murate of Potash (60\% $\mathrm{K}_{2} \mathrm{O}$ ), respectively. Other agronomic practices were conducted according to recommended for rice cultivation in Nepal.

\section{Data collection and analysis}

Observations were recorded on days to $50 \%$ flowering, days to $80 \%$ maturity, plant height $(\mathrm{cm})$, panicle length $(\mathrm{cm})$ grain yield
( $\mathrm{kg} / \mathrm{ha}$ ) and straw yield ( $\mathrm{kg} / \mathrm{ha}$ ). For statistical analysis, analysis of variance among traits were done by using STAR package at 0.05 level of significance. Likewise, Daily maximum temperature, minimum temperature and rainfall were recorded during rice growing season (Figure 1).

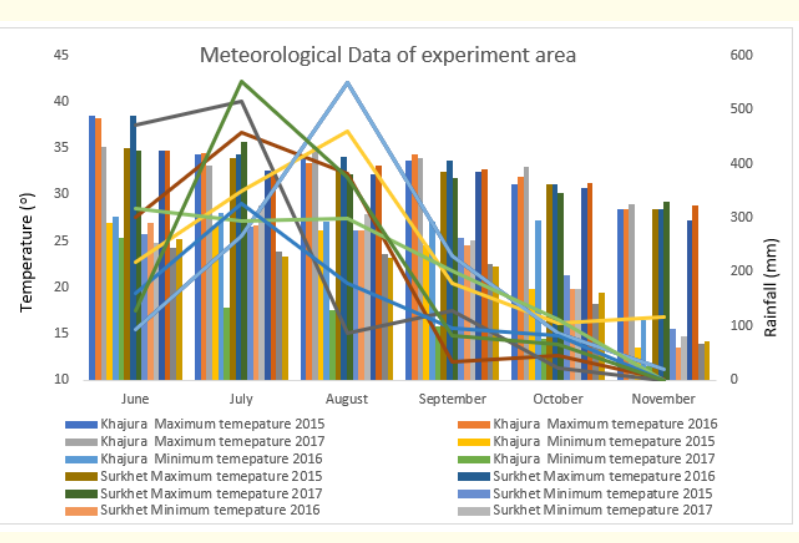

Figure 1: Meteorological situation of experimental areas.

\section{Result and Discussion}

Days to flowering

The pooled analysis of data revealed that the genotypes were highly significant for days to $50 \%$ flowering in all location (Table 1 ). Days to $50 \%$ flowering ranged from 83 days after sowing (IR 83376-B-B-71-1) to 93 DAS (IR 95840-33-3-2-1) in stress in reproductive stage where as in normal irrigated condition (control) days to flowering was ranged from 80 days (IR 87754-42-1-3-3) to 88 days IR 87761-39-1-1-4 and IR 95840-33-3-2-1) at RARS, Khajura. The genotype IR 83376-B-B-71-1 and IR 80461-B-79-3 flowered same day in both irrigated and reproductive stress condition. So, we concluded that these varieties were tolerance of drought. Likewise, genotype IR 80461-B-79-3 flowered early i.e.91 days in reproductive stress condition and 88 days in control and genotype IR 79615-9-3-1-3 flowered early i.e. 100 days in reproductive stress condition and 97 days in control at ARS, Surkhet. Similarly, Days to $50 \%$ flowering ranged from after sowing 84 days (IR 80461-B-793) to 97 DAS (IR 79615-9-3-1-3) at RARS, Doti. In drought condition, days to flowering delayed due to decrease of water potential in plant tissues generate high moisture tension in plant which inhibits flower primordia development [13]. 


\begin{tabular}{|c|c|c|c|c|c|c|c|c|c|c|c|c|c|c|c|c|}
\hline \multirow{3}{*}{ Genotypes } & \multicolumn{8}{|c|}{ RARS Khajura } & \multirow{2}{*}{\multicolumn{4}{|c|}{ ARS, Surkhet }} & \multirow{2}{*}{\multicolumn{4}{|c|}{ RARS, Doti }} \\
\hline & \multicolumn{4}{|c|}{ Irrigated } & \multicolumn{4}{|c|}{ Reproductive stress } & & & & & & & & \\
\hline & 2015 & 2016 & 2017 & Mean & 2015 & 2016 & 2017 & Mean & 2015 & 2016 & 2017 & Mean & 2015 & 2016 & 2017 & Mean \\
\hline IR 83376-B-B-91-3 & 87 & 86 & 83 & 85 & 83 & 93 & 88 & 88 & 88 & 94 & 90 & 91 & 87 & 99 & 90 & 92 \\
\hline IR 82608-B-B-33-2 & 82 & 86 & 81 & 83 & 81 & 89 & 88 & 86 & 90 & 95 & 91 & 92 & 88 & 99 & 91 & 93 \\
\hline IR 74371-70-1-1 & 83 & 86 & 82 & 84 & 83 & 91 & 88 & 87 & 90 & 98 & 96 & 95 & 89 & 99 & 96 & 95 \\
\hline IR 87754-42-2-2-4 & 83 & 88 & 81 & 84 & 83 & 92 & 91 & 89 & 89 & 92 & 92 & 91 & 91 & 93 & 92 & 92 \\
\hline IR 87759-12-2-1-1 & 83 & 88 & 85 & 85 & 84 & 93 & 89 & 89 & 88 & 95 & 91 & 91 & 89 & 99 & 91 & 93 \\
\hline IR 87753-11-2-1-1 & 84 & 87 & 82 & 84 & 86 & 90 & 90 & 89 & 87 & 94 & 93 & 91 & 86 & 95 & 93 & 91 \\
\hline IR 87761-51-1-1-4 & 83 & 87 & 80 & 83 & 84 & 87 & 88 & 86 & 89 & 93 & 92 & 91 & 86 & 95 & 92 & 91 \\
\hline IR 83383-B-B-141-1 & 83 & 85 & 81 & 83 & 83 & 89 & 89 & 87 & 88 & 93 & 92 & 91 & 86 & 95 & 92 & 91 \\
\hline IR 84859-B-86-3-1 & 84 & 85 & 79 & 83 & 79 & 89 & 89 & 86 & 91 & 93 & 89 & 91 & 86 & 97 & 89 & 91 \\
\hline IR 87761-52-1-2-2 & 82 & 88 & 80 & 83 & 80 & 92 & 88 & 87 & 92 & 92 & 90 & 91 & 87 & 95 & 90 & 91 \\
\hline IR 87761-39-1-1-4 & 90 & 88 & 86 & 88 & 93 & 93 & 90 & 92 & 91 & 98 & 98 & 96 & 87 & 98 & 98 & 94 \\
\hline IR 87751-20-4-4-2 & 84 & 88 & 82 & 85 & 83 & 92 & 89 & 88 & 89 & 93 & 91 & 91 & 88 & 96 & 91 & 92 \\
\hline IR 87754-42-1-3-3 & 74 & 87 & 80 & 80 & 84 & 91 & 88 & 88 & 85 & 95 & 90 & 90 & 85 & 100 & 90 & 92 \\
\hline IR 87749-10-1-1-4 & 87 & 88 & 82 & 86 & 84 & 92 & 88 & 88 & 87 & 94 & 92 & 91 & 88 & 96 & 92 & 92 \\
\hline Sarju-52 & 84 & 89 & 86 & 86 & 85 & 87 & 92 & 88 & 96 & 97 & 90 & 94 & 99 & 103 & 90 & 97 \\
\hline IR 83376-B-B-71-1 & 82 & 84 & 83 & 83 & 79 & 84 & 87 & 83 & 85 & 95 & 92 & 91 & 86 & 98 & 92 & 92 \\
\hline IR 80461-B-79-3 & 81 & 90 & 83 & 85 & 77 & 94 & 84 & 85 & 85 & 91 & 88 & 88 & 84 & 94 & 88 & 89 \\
\hline IR 95781-15-1-1-4 & 83 & 86 & 87 & 85 & 82 & 88 & 87 & 86 & 87 & 92 & 89 & 89 & 86 & 95 & 89 & 90 \\
\hline IR 78875-207-B-3-B & 87 & 84 & 83 & 85 & 88 & 88 & 92 & 89 & 89 & 95 & 93 & 92 & 88 & 97 & 93 & 93 \\
\hline IR 95840-33-3-2-1 & 89 & 89 & 87 & 88 & 90 & 93 & 94 & 92 & 92 & 97 & 92 & 94 & 95 & 101 & 92 & 96 \\
\hline IR 89889-18-1-2-1 & 84 & 87 & 84 & 85 & 83 & 91 & 94 & 89 & 92 & 97 & 93 & 94 & 92 & 101 & 93 & 95 \\
\hline IR 93809-2-1-3-2 & 83 & 85 & 81 & 83 & 84 & 87 & 87 & 86 & 92 & 93 & 91 & 92 & 90 & 95 & 91 & 92 \\
\hline IR 95814-29-1-1-3 & 88 & 86 & 88 & 87 & 91 & 88 & 94 & 91 & 98 & 100 & 97 & 98 & 97 & 103 & 97 & 99 \\
\hline Sukhkha Dhan-2 & 83 & 84 & 82 & 83 & 79 & 88 & 86 & 84 & 85 & 93 & 89 & 89 & 86 & 96 & 89 & 90 \\
\hline F-test & $*$ & $* *$ & $* *$ & ** & $* *$ & $* *$ & $* *$ & $* *$ & $* *$ & ** & $* *$ & $* *$ & ** & ** & $* *$ & $*$ \\
\hline LSD & 7.43 & 2.88 & 2.30 & 4.20 & 2.73 & 2.88 & 4.10 & 3.24 & 4.10 & 2.20 & 2.60 & 3.20 & 3.80 & 1.3 & 2.60 & 2.57 \\
\hline $\mathrm{CV}$ & 5.36 & 2.02 & 2.00 & 4.01 & 1.99 & 1.95 & 2.7 & 6.64 & 2.80 & 9.20 & 1.40 & 8.20 & 2.10 & 3.80 & 1.40 & 7.30 \\
\hline
\end{tabular}

Table 1: Day to 50\% flowering of drought tolerant rice genotypes over the location in different moisture condition during 2015 to 2017.

Plant height

Plant height was highly affected by moisture stress. In Normal irrigated condition at Khajura condition, all genotypes grow well and had taller plant. Likewise, shorter plant was found in reproductive stress condition. In 2017, rainfall was well distributed in Khajura, so taller plant was found in both irrigated and reproductive stress condition. In rainfed condition, plant did not suffer on early stage and suffer in later stage so, plant height affected less than drought condition. Plant height reduce under water stress condition because of plant development is reduced as a consequence of poor root development by impaired mitosis; cell elongation and expansion; reduced leaf-surface traits (form, shape, composition of cuticular and epicuticular wax, leaf pubescence and leaf color), which affect the radiation load on the leaf canopy and inhibition of stem reserves [14,15]. From these, we concluded plant height was more responsive in early moisture stress than later stage. Detail result is presented in table 2. 


\begin{tabular}{|c|c|c|c|c|c|c|c|c|c|c|c|c|c|c|c|c|}
\hline \multirow{3}{*}{ Genotypes } & \multicolumn{8}{|c|}{ RARS Khajura } & \multirow{2}{*}{\multicolumn{4}{|c|}{ ARS, Surkhet }} & \multirow{2}{*}{\multicolumn{4}{|c|}{ RARS, Doti }} \\
\hline & \multicolumn{4}{|c|}{ Irrigated } & \multicolumn{4}{|c|}{\begin{tabular}{|l|} 
Reproductive stress \\
\end{tabular}} & & & & & & & & \\
\hline & 2015 & 2016 & 2017 & Mean & 2015 & 2016 & 2017 & Mean & 2015 & 2016 & 2017 & Mean & 2015 & 2016 & 2017 & Mean \\
\hline IR 83376-B-B-91-3 & 94 & 101 & 106 & 100 & 79 & 66 & 88 & 88 & 97 & 80 & 88 & 88 & 81 & 99 & 95 & 92 \\
\hline IR 82608-B-B-33-2 & 102 & 106 & 108 & 105 & 81 & 72 & 92 & 86 & 108 & 97 & 102 & 102 & 75 & 99 & 93 & 89 \\
\hline IR 74371-70-1-1 & 99 & 103 & 112 & 105 & 68 & 75 & 87 & 87 & 105 & 84 & 95 & 95 & 79 & 99 & 105 & 94 \\
\hline IR $87754-42-2-2-4$ & 99 & 102 & 103 & 101 & 83 & 72 & 83 & 89 & 86 & 78 & 82 & 82 & 74 & 93 & 97 & 88 \\
\hline IR 87759-12-2-1-1 & 102 & 105 & 97 & 101 & 80 & 71 & 88 & 89 & 92 & 82 & 87 & 87 & 73 & 99 & 98 & 90 \\
\hline IR 87753-11-2-1-1 & 98 & 103 & 105 & 102 & 75 & 73 & 88 & 89 & 78 & 78 & 78 & 78 & 71 & 95 & 100 & 89 \\
\hline IR 87761-51-1-1-4 & 96 & 101 & 106 & 101 & 76 & 75 & 93 & 86 & 85 & 79 & 82 & 82 & 76 & 95 & 100 & 90 \\
\hline IR 83383-B-B-141-1 & 98 & 102 & 104 & 101 & 76 & 71 & 94 & 87 & 86 & 91 & 88 & 88 & 76 & 95 & 92 & 88 \\
\hline IR 84859-B-86-3-1 & 92 & 94 & 102 & 96 & 77 & 78 & 84 & 86 & 85 & 81 & 83 & 83 & 72 & 97 & 95 & 88 \\
\hline IR 87761-52-1-2-2 & 98 & 104 & 104 & 102 & 79 & 77 & 90 & 87 & 87 & 78 & 82 & 82 & 71 & 95 & 101 & 89 \\
\hline IR 87761-39-1-1-4 & 99 & 102 & 105 & 102 & 73 & 68 & 79 & 92 & 86 & 73 & 79 & 79 & 76 & 98 & 90 & 88 \\
\hline IR 87751-20-4-4-2 & 96 & 103 & 100 & 100 & 78 & 63 & 91 & 88 & 91 & 81 & 86 & 86 & 74 & 96 & 98 & 89 \\
\hline IR 87754-42-1-3-3 & 93 & 104 & 102 & 100 & 72 & 78 & 90 & 88 & 88 & 79 & 83 & 83 & 75 & 100 & 94 & 90 \\
\hline IR 87749-10-1-1-4 & 94 & 104 & 103 & 100 & 73 & 78 & 90 & 88 & 95 & 80 & 88 & 88 & 79 & 96 & 96 & 90 \\
\hline Sarju-52 & 99 & 94 & 100 & 98 & 75 & 69 & 90 & 88 & 83 & 73 & 78 & 78 & 65 & 103 & 85 & 84 \\
\hline IR 83376-B-B-71-1 & 98 & 109 & 98 & 102 & 78 & 73 & 90 & 83 & 76 & 80 & 78 & 78 & 70 & 98 & 92 & 87 \\
\hline IR 80461-B-79-3 & 98 & 105 & 105 & 103 & 90 & 68 & 92 & 85 & 99 & 92 & 95 & 95 & 76 & 94 & 97 & 89 \\
\hline IR 95781-15-1-1-4 & 103 & 110 & 112 & 108 & 73 & 77 & 101 & 86 & 107 & 97 & 102 & 102 & 87 & 95 & 96 & 93 \\
\hline IR 78875-207-B-3-B & 99 & 103 & 103 & 102 & 77 & 74 & 94 & 89 & 90 & 88 & 89 & 89 & 80 & 97 & 98 & 92 \\
\hline IR 95840-33-3-2-1 & 113 & 82 & 112 & 102 & 87 & 62 & 104 & 92 & 120 & 117 & 118 & 118 & 89 & 101 & 110 & 100 \\
\hline IR 89889-18-1-2-1 & 95 & 90 & 105 & 97 & 79 & 67 & 92 & 89 & 98 & 86 & 92 & 92 & 81 & 101 & 93 & 92 \\
\hline IR 93809-2-1-3-2 & 100 & 89 & 103 & 97 & 81 & 64 & 91 & 86 & 98 & 83 & 91 & 91 & 80 & 95 & 92 & 89 \\
\hline IR 95814-29-1-1-3 & 106 & 87 & 110 & 101 & 82 & 65 & 94 & 91 & 88 & 88 & 88 & 88 & 72 & 103 & 94 & 90 \\
\hline Sukhkha Dhan-2 & 105 & 105 & 98 & 103 & 86 & 77 & 91 & 84 & 92 & 94 & 93 & 93 & 75 & 96 & 100 & 90 \\
\hline F-test & $*$ & $* *$ & $* *$ & $* *$ & $*$ & $*$ & $*$ & $* *$ & $* *$ & $* *$ & $* *$ & $* *$ & $* *$ & $* *$ & NS & $*$ \\
\hline LSD & 9.08 & 9.5 & 3.71 & 7.44 & 12.7 & 9.9 & 9.20 & 10.6 & 8.5 & 9.1 & 14. & 3.2 & 6.69 & 1.3 & & 4.0 \\
\hline $\mathrm{CV}$ & 5.59 & 5.7 & 9.20 & 4.01 & 9.89 & 8.4 & 12.3 & 12.1 & 5.6 & 5.2 & 9.1 & 8.2 & 5.36 & 3.8 & 7.34 & 9.6 \\
\hline
\end{tabular}

Table 2: Plant height (cm) of drought tolerant rice genotypes over the location in different moisture condition during 2015 to 2017.

Panicle length

Long panicles were found in Surkhet than other places. Likewise, in normal irrigated condition all genotypes bared long panicle and (table 3). In drought condition all genotypes bared short panicle than normal irrigated and rainfed condition. In water shortage condition, decrease in translocation of assimilates towards reproductive organs which ultimately decreased flower organ formation [16]. Likewise, genotypes IR 87754-421-3-3, IR 89889-18-1-2-1 and IR 80461-B-79-3 had less affected by water stress in term of panicle length than other genotypes. Detailed result is presented in table 3. 


\begin{tabular}{|c|c|c|c|c|c|c|c|c|c|c|c|c|c|c|c|c|}
\hline \multirow{3}{*}{ Genotypes } & \multicolumn{8}{|c|}{ RARS Khajura } & \multirow{2}{*}{\multicolumn{4}{|c|}{ ARS, Surkhet }} & \multirow{2}{*}{\multicolumn{4}{|c|}{ RARS, Doti }} \\
\hline & \multicolumn{4}{|c|}{ Irrigated } & \multicolumn{4}{|c|}{ Reproductive stress } & & & & & & & & \\
\hline & 2015 & 2016 & 2017 & Mean & 2015 & 2016 & 2017 & Mean & 2015 & 2016 & 2017 & Mean & 2015 & 2016 & 2017 & Mean \\
\hline IR 83376-B-B-91-3 & 23 & 28 & 26 & 26 & 19 & 18 & 20 & 19 & 26 & 29 & 27 & 27 & 25 & 22 & 24 & 23 \\
\hline IR 82608-B-B-33-2 & 24 & 25 & 25 & 25 & 20 & 18 & 21 & 20 & 28 & 29 & 28 & 28 & 23 & 22 & 26 & 24 \\
\hline IR 74371-70-1-1 & 25 & 28 & 26 & 26 & 18 & 22 & 21 & 20 & 29 & 27 & 28 & 28 & 25 & 25 & 24 & 25 \\
\hline IR $87754-42-2-2-4$ & 29 & 26 & 26 & 27 & 22 & 21 & 22 & 22 & 29 & 26 & 27 & 27 & 25 & 26 & 25 & 25 \\
\hline IR 87759-12-2-1-1 & 29 & 25 & 25 & 26 & 23 & 19 & 20 & 21 & 27 & 29 & 28 & 28 & 25 & 26 & 27 & 26 \\
\hline IR 87753-11-2-1-1 & 26 & 27 & 26 & 26 & 23 & 21 & 22 & 22 & 28 & 27 & 29 & 28 & 25 & 26 & 27 & 26 \\
\hline IR 87761-51-1-1-4 & 26 & 24 & 26 & 25 & 22 & 20 & 21 & 21 & 29 & 27 & 29 & 28 & 26 & 26 & 28 & 27 \\
\hline IR 83383-B-B-141-1 & 24 & 26 & 27 & 26 & 20 & 22 & 20 & 21 & 28 & 27 & 28 & 28 & 22 & 25 & 25 & 24 \\
\hline IR 84859-B-86-3-1 & 27 & 25 & 23 & 25 & 19 & 22 & 20 & 20 & 28 & 26 & 27 & 27 & 20 & 24 & 26 & 23 \\
\hline IR 87761-52-1-2-2 & 28 & 30 & 25 & 27 & 22 & 23 & 24 & 23 & 29 & 28 & 29 & 29 & 24 & 25 & 27 & 25 \\
\hline IR 87761-39-1-1-4 & 28 & 29 & 24 & 27 & 21 & 21 & 21 & 21 & 24 & 26 & 25 & 25 & 20 & 21 & 23 & 21 \\
\hline IR 87751-20-4-4-2 & 24 & 29 & 26 & 26 & 21 & 18 & 23 & 21 & 26 & 27 & 27 & 27 & 25 & 24 & 27 & 25 \\
\hline IR 87754-42-1-3-3 & 22 & 26 & 27 & 25 & 20 & 21 & 24 & 22 & 27 & 28 & 28 & 28 & 24 & 26 & 26 & 25 \\
\hline IR 87749-10-1-1-4 & 21 & 27 & 27 & 25 & 21 & 23 & 23 & 22 & 27 & 30 & 29 & 28 & 24 & 23 & 28 & 25 \\
\hline Sarju-52 & 26 & 22 & 26 & 25 & 21 & 19 & 21 & 20 & 26 & 29 & 28 & 28 & 24 & 24 & 26 & 24 \\
\hline IR 83376-B-B-71-1 & 25 & 25 & 21 & 24 & 19 & 21 & 19 & 20 & 25 & 26 & 25 & 25 & 23 & 19 & 23 & 22 \\
\hline IR 80461-B-79-3 & 25 & 22 & 26 & 24 & 23 & 19 & 23 & 22 & 26 & 28 & 27 & 27 & 24 & 22 & 27 & 24 \\
\hline IR 95781-15-1-1-4 & 27 & 23 & 24 & 25 & 19 & 23 & 20 & 21 & 26 & 27 & 27 & 26 & 21 & 23 & 26 & 23 \\
\hline IR 78875-207-B-3-B & 25 & 26 & 23 & 25 & 22 & 23 & 20 & 21 & 23 & 25 & 24 & 24 & 23 & 22 & 24 & 23 \\
\hline IR 95840-33-3-2-1 & 24 & 19 & 22 & 22 & 19 & 17 & 21 & 19 & 24 & 27 & 26 & 25 & 19 & 22 & 23 & 21 \\
\hline IR 89889-18-1-2-1 & 26 & 22 & 25 & 24 & 22 & 22 & 23 & 22 & 27 & 27 & 27 & 27 & 23 & 21 & 24 & 23 \\
\hline IR 93809-2-1-3-2 & 24 & 23 & 26 & 24 & 19 & 17 & 20 & 19 & 28 & 26 & 28 & 27 & 22 & 23 & 25 & 23 \\
\hline IR 95814-29-1-1-3 & 26 & 26 & 26 & 26 & 23 & 17 & 24 & 21 & 30 & 26 & 28 & 28 & 20 & 24 & 26 & 23 \\
\hline Sukhkha Dhan-2 & 26 & 23 & 22 & 24 & 20 & 20 & 19 & 20 & 23 & 26 & 25 & 25 & 21 & 21 & 23 & 22 \\
\hline F-test & NS & $* *$ & $* *$ & NS & NS & $*$ & NS & NS & $* *$ & NS & $* *$ & NS & $* *$ & NS & NS & NS \\
\hline LSD & & 3.4 & 2.53 & & & 5.4 & & & 2.2 & & 1.9 & & 2.99 & & & \\
\hline $\mathrm{CV}$ & 14.6 & 8.3 & 13.6 & 14.0 & 13.3 & 9.6 & 12.3 & 12.1 & 7.5 & 5.8 & 8.6 & 8.2 & 7.90 & 6.1 & 7.50 & 9.7 \\
\hline
\end{tabular}

Table 3: Panicle Length $(\mathrm{cm})$ of drought tolerant rice genotypes over the location in different moisture condition during 2015 to 2017.

Grain yield

Highest grain yield was obtained in irrigated trial than rainfed and reproductive drought trial. In 2016, trials suffered from more water stress than other two years in drought and rainfed trial which decreased grain yield (table 4). Among the location, higher grain yield found in Surkhet than Doti in rainfed condition. In 2015, higher and well distribute rainfall in Surkhet and rice received sufficient water and produced higher grain yield than irrigated. In irrigated trail during 2015, heavy rainfall just after transplanting, killed seedling and gap filling done after 15 days reduced tillering and produced lower grain yield. Likewise, in 2017, rainfall is well distributed and optimum amount during vegetative stage which increase grain yield in all location. In water stress condition reduced of water content, diminished leaf water potential, turgor pressure, stomatal activity and decreased in cell enlargement and growth which affect various physiological and biochemical processes, such as photosynthesis, respiration, translocation, ion uptake, carbohydrates, nutrient metabolism and growth promoters and decrease net assimilation [17]. Detail is presented in table 4.

Citation: Santosh Raj Tripath., et al. "Evaluation of Drought Tolerant Rice Genotypes in Different Moisture Regime on Western Nepal". Acta Scientific Agriculture 3.11 (2019): 100-108. 


\begin{tabular}{|c|c|c|c|c|c|c|c|c|c|c|c|c|c|c|c|c|}
\hline \multirow{3}{*}{ Genotypes } & \multicolumn{8}{|c|}{ RARS Khajura } & \multirow{2}{*}{\multicolumn{4}{|c|}{ ARS, Surkhet }} & \multirow{2}{*}{\multicolumn{4}{|c|}{ RARS, Doti }} \\
\hline & \multicolumn{4}{|c|}{ Irrigated } & \multicolumn{4}{|c|}{ Reproductive stress } & & & & & & & & \\
\hline & 2015 & 2016 & 2017 & Mean & 2015 & 2016 & 2017 & Mean & 2015 & 2016 & 2017 & Mean & 2015 & 2016 & 2017 & Mean \\
\hline IR 83376-B-B-91-3 & 3417 & 4757 & 4748 & 4307 & 2183 & 602 & 2908 & 1898 & 4191 & 3710 & 3960 & 3954 & 3553 & 2819 & 3883 & 3419 \\
\hline IR 82608-B-B-33-2 & 3286 & 5161 & 4397 & 4281 & 1700 & 1854 & 2626 & 2060 & 4222 & 4299 & 4135 & 4219 & 2309 & 3056 & 3050 & 2805 \\
\hline IR 74371-70-1-1 & 3699 & 4268 & 5165 & 4377 & 1221 & 568 & 2454 & 1414 & 4594 & 3372 & 3912 & 3959 & 4198 & 2653 & 4967 & 3939 \\
\hline IR 87754-42-2-2-4 & 3807 & 4362 & 4691 & 4287 & 1630 & 609 & 3348 & 1862 & 3957 & 4296 & 4569 & 4274 & 4399 & 4444 & 3933 & 4259 \\
\hline IR 87759-12-2-1-1 & 3592 & 5009 & 4431 & 4344 & 1841 & 873 & 2574 & 1763 & 4785 & 3643 & 4156 & 4194 & 3496 & 4236 & 4217 & 3983 \\
\hline IR 87753-11-2-1-1 & 3225 & 4598 & 4395 & 4073 & 750 & 801 & 2485 & 1345 & 3545 & 4244 & 4155 & 3981 & 4439 & 3549 & 3533 & 3840 \\
\hline IR 87761-51-1-1-4 & 4197 & 5512 & 4690 & 4800 & 2187 & 727 & 2578 & 1831 & 4694 & 4354 & 4557 & 4535 & 3634 & 3889 & 4050 & 3858 \\
\hline IR 83383-B-B-141-1 & 3316 & 4880 & 4885 & 4360 & 1697 & 1223 & 2565 & 1828 & 3872 & 3832 & 3907 & 3870 & 1765 & 3528 & 2967 & 2753 \\
\hline IR 84859-B-86-3-1 & 3984 & 4975 & 4043 & 4334 & 1897 & 639 & 1924 & 1487 & 4448 & 3453 & 3904 & 3935 & 3447 & 3840 & 5033 & 4107 \\
\hline IR 87761-52-1-2-2 & 4192 & 4925 & 4176 & 4431 & 2603 & 2381 & 1968 & 2317 & 4469 & 3846 & 4154 & 4156 & 3279 & 4146 & 4617 & 4014 \\
\hline IR 87761-39-1-1-4 & 4172 & 4274 & 6204 & 4883 & 2381 & 549 & 2505 & 1812 & 4469 & 3731 & 4083 & 4094 & 2024 & 3354 & 4883 & 3420 \\
\hline IR 87751-20-4-4-2 & 3889 & 4488 & 4731 & 4369 & 1632 & 675 & 2439 & 1582 & 4080 & 3778 & 3957 & 3938 & 3835 & 3368 & 2633 & 3279 \\
\hline IR $87754-42-1-3-3$ & 3799 & 5262 & 5114 & 4725 & 1499 & 825 & 2980 & 1768 & 4642 & 3602 & 4074 & 4106 & 2479 & 3715 & 4417 & 3537 \\
\hline IR $87749-10-1-1-4$ & 3407 & 4231 & 4738 & 4125 & 1694 & 656 & 2540 & 1630 & 3917 & 3814 & 4245 & 3992 & 2749 & 3681 & 4283 & 3571 \\
\hline Sarju-52 & 3481 & 4808 & 5038 & 4442 & 1494 & 1487 & 2125 & 1702 & 3785 & 4090 & 4431 & 4102 & 2706 & 2167 & 4467 & 3113 \\
\hline IR 83376-B-B-71-1 & 3354 & 5228 & 4609 & 4397 & 3056 & 3516 & 3196 & 3256 & 3924 & 3792 & 3903 & 3873 & 3378 & 3194 & 3767 & 3446 \\
\hline IR 80461-B-79-3 & 3202 & 6034 & 4459 & 4565 & 2462 & 706 & 2735 & 1968 & 3920 & 3216 & 3547 & 3561 & 3405 & 2403 & 4167 & 3325 \\
\hline IR 95781-15-1-1-4 & 3636 & 5623 & 4716 & 4658 & 1095 & 608 & 2380 & 1361 & 4747 & 3880 & 4286 & 4304 & 3850 & 1875 & 3983 & 3236 \\
\hline IR 78875-207-B-3-B & 3828 & 5253 & 4706 & 4596 & 1094 & 959 & 2842 & 1632 & 4479 & 3893 & 4188 & 4187 & 4259 & 3521 & 4233 & 4004 \\
\hline IR 95840-33-3-2-1 & 3793 & 4414 & 5028 & 4412 & 1290 & 3368 & 2051 & 2236 & 4406 & 3927 & 4179 & 4171 & 2305 & 1625 & 3483 & 2471 \\
\hline IR 89889-18-1-2-1 & 3654 & 4755 & 4616 & 4342 & 1564 & 493 & 2590 & 1549 & 4073 & 4394 & 4333 & 4267 & 4119 & 1771 & 3967 & 3285 \\
\hline IR 93809-2-1-3-2 & 3389 & 4742 & 3615 & 3915 & 1773 & 1136 & 1980 & 1630 & 4038 & 3643 & 3857 & 3846 & 3047 & 2569 & 3433 & 3016 \\
\hline IR 95814-29-1-1-3 & 3292 & 4524 & 5039 & 4285 & 1089 & 763 & 1418 & 1090 & 3906 & 3785 & 3892 & 3861 & 1301 & 1576 & 3667 & 2181 \\
\hline Sukhkha Dhan-2 & 3935 & 4925 & 4998 & 4619 & 1362 & 775 & 2967 & 1701 & 3986 & 3981 & 4044 & 4004 & 2689 & 2139 & 4083 & 2970 \\
\hline Mean & 3648 & 4875 & 4718 & 4414 & 1716 & 1116 & 2507 & 1780 & 4215 & 3857 & 4101 & 4058 & 3194 & 3047 & 3988 & 3410 \\
\hline F-test & NS & $* *$ & $*$ & $* *$ & $*$ & $* *$ & $*$ & $* *$ & $*$ & NS & NS & $* *$ & $* *$ & $* *$ & $* *$ & $*$ \\
\hline LSD & & 858 & 1837 & 1348 & 1488 & 563 & 892 & 981 & 571 & & & 1271 & 1736 & 1353 & 1900 & 1663 \\
\hline $\mathrm{CV}$ & 18.52 & 10.7 & 18.7 & 19.01 & 22.75 & 20.7 & 21.7 & 22.10 & 10.26 & 11.5 & 18.9 & 18.20 & 12.66 & 16.7 & 10.66 & 19.7 \\
\hline
\end{tabular}

Table 4: Grain yield (kg/ha) of drought tolerant rice genotypes over the location in different moisture condition during 2015 to 2017.

Higher grain yield reduction was found in reproductive stage drought $(58.9 \%)$ than normal irrigated condition. Likewise, grain yield reduction $7.9 \%$ at Surkhet and 22.7 at Doti in rainfed condition. Similarly, genotypes IR 74371-70-1-1, IR 87753-11-2-1-1 and IR 84859-B-86-3-1 were highly susceptible to water stress and reduced more than $65 \%$ grain yield in reproductive drought than normal irrigated condition. Likewise, genotypes IR 95814-29-1-1-3, IR 95840-33-3-2-1, IR 80461-B-79-3, Sukhkha Dhan-
2, IR 83383-B-B-141-1 and IR 87761-39-1-1-4 had found higher grain yield reduction in rainfed condition than normal irrigated condition. Yield reduction in water stress is due to reduction of cell water content, diminished leaf water potential, turgor pressure, stomatal activity and decrease in cell enlargement and growth. Severe water stress may result in the arrest of photosynthesis, disturbance in metabolism [18] (Table 5). 
Evaluation of Drought Tolerant Rice Genotypes in Different Moisture Regime on Western Nepal

\begin{tabular}{|c|c|c|c|c|}
\hline \multirow{2}{*}{ EN } & \multirow{2}{*}{ Genotypes } & \multicolumn{3}{|c|}{ Grain yield reduction \% } \\
\hline & & Reproductive Stress & Rainfed Surkhet & Rainfed Doti \\
\hline 1 & IR 83376-B-B-91-3 & 55.9 & 8.2 & 20.6 \\
\hline 2 & IR 82608-B-B-33-2 & 51.9 & 1.5 & 34.5 \\
\hline 3 & IR 74371-70-1-1 & 67.7 & 9.5 & 10.0 \\
\hline 4 & IR 87754-42-2-2-4 & 56.6 & 0.3 & 0.6 \\
\hline 5 & IR 87759-12-2-1-1 & 59.4 & 3.4 & 8.3 \\
\hline 6 & IR 87753-11-2-1-1 & 67.0 & 2.2 & 5.7 \\
\hline 7 & IR 87761-51-1-1-4 & 61.9 & 5.5 & 19.6 \\
\hline 8 & IR 83383-B-B-141-1 & 58.1 & 11.2 & 36.9 \\
\hline 9 & IR 84859-B-86-3-1 & 65.7 & 9.2 & 5.2 \\
\hline 10 & IR 87761-52-1-2-2 & 47.7 & 6.2 & 9.4 \\
\hline 11 & IR 87761-39-1-1-4 & 62.9 & 16.2 & 30.0 \\
\hline 12 & IR 87751-20-4-4-2 & 63.8 & 9.9 & 25.0 \\
\hline 13 & IR 87754-42-1-3-3 & 62.6 & 13.1 & 25.1 \\
\hline 14 & IR 87749-10-1-1-4 & 60.5 & 3.2 & 13.4 \\
\hline 15 & Sarju-52 & 61.7 & 7.7 & 29.9 \\
\hline 16 & IR 83376-B-B-71-1 & 26.0 & 11.9 & 21.6 \\
\hline 17 & IR 80461-B-79-3 & 56.9 & 22.0 & 27.2 \\
\hline 18 & IR 95781-15-1-1-4 & 70.8 & 7.6 & 30.5 \\
\hline 19 & IR 78875-207-B-3-B & 64.5 & 8.9 & 12.9 \\
\hline 20 & IR 95840-33-3-2-1 & 49.3 & 5.5 & 44.0 \\
\hline 21 & IR 89889-18-1-2-1 & 64.3 & 1.7 & 24.3 \\
\hline 22 & IR 93809-2-1-3-2 & 58.4 & 1.8 & 23.0 \\
\hline 23 & IR 95814-29-1-1-3 & 74.6 & 9.9 & 49.1 \\
\hline \multirow[t]{2}{*}{24} & Sukhkha Dhan-2 & 63.2 & 13.3 & 35.7 \\
\hline & Mean & 59.8 & 7.9 & 22.7 \\
\hline
\end{tabular}

Table 5: Mean Grain yield reduction percentage in drought and rainfed with respect to normal irrigated.

Straw yield

Straw yield was higher in normal irrigated than reproductive drought and rainfed condition due to higher plant height and less tiller motility in irrigated condition. In drought condition, plant suffered from water stress from tillering stage, so plant growth stunt and reduced tillering. Likewise, in rainfed condition, early growth is better and higher tiller but later stage when water become limited then young tiller found dead. Water is a major constituent of plant tissue as reagent for chemical reactions and solvent for translocation of metabolites and minerals as well as an essential component for cell enlargement through increasing turgor pressure. When water stress arises then plant check to translocation of metabolites to young parts than young plant parts died [19]. Among the genotypes IR 95840-33-3-2-1, IR 95814-29-1-1-3 and IR 87753-11-2-1-1 had produced higher straw yield (Table 6). 
Evaluation of Drought Tolerant Rice Genotypes in Different Moisture Regime on Western Nepal

\begin{tabular}{|c|c|c|c|c|c|c|c|c|c|c|c|c|}
\hline \multirow{3}{*}{ Genotypes } & \multicolumn{8}{|c|}{ RARS Khajura } & \multirow{2}{*}{\multicolumn{4}{|c|}{ ARS, Surkhet }} \\
\hline & \multicolumn{4}{|c|}{ Irrigated } & \multicolumn{4}{|c|}{ Reproductive stress } & & & & \\
\hline & 2015 & 2016 & 2017 & Mean & 2015 & 2016 & 2017 & Mean & 2015 & 2016 & 2017 & Mean \\
\hline IR 83376-B-B-91-3 & 6761 & 5283 & 4950 & 5664 & 3303 & 1598 & 2900 & 2600 & 4988 & 3589 & 4555 & 4377 \\
\hline IR 82608-B-B-33-2 & 5087 & 4703 & 4750 & 4847 & 4133 & 2570 & 2867 & 3190 & 4599 & 5044 & 5268 & 4970 \\
\hline IR 74371-70-1-1 & 4674 & 7587 & 4750 & 5670 & 3640 & 2431 & 2733 & 2935 & 4708 & 3914 & 4624 & 4415 \\
\hline IR 87754-42-2-2-4 & 5218 & 4438 & 4500 & 4719 & 3301 & 2431 & 3133 & 2955 & 5395 & 4449 & 5276 & 5040 \\
\hline IR 87759-12-2-1-1 & 5744 & 5659 & 4650 & 5351 & 3853 & 2223 & 3067 & 3048 & 5368 & 3541 & 4705 & 4538 \\
\hline IR 87753-11-2-1-1 & 4664 & 7071 & 4200 & 5312 & 4319 & 2917 & 2400 & 3212 & 5027 & 4713 & 5266 & 5002 \\
\hline IR 87761-51-1-1-4 & 5446 & 3933 & 4750 & 4710 & 3994 & 2152 & 2133 & 2760 & 5173 & 4157 & 4993 & 4774 \\
\hline IR 83383-B-B-141-1 & 5378 & 4470 & 4950 & 4933 & 4484 & 2014 & 2667 & 3055 & 4633 & 4550 & 4981 & 4721 \\
\hline IR 84859-B-86-3-1 & 5501 & 6042 & 4000 & 5181 & 3381 & 2709 & 2667 & 2919 & 4189 & 3514 & 4134 & 3945 \\
\hline IR 87761-52-1-2-2 & 5398 & 4904 & 4750 & 5017 & 2883 & 3473 & 2767 & 3041 & 4622 & 4049 & 4666 & 4446 \\
\hline IR 87761-39-1-1-4 & 5153 & 5693 & 5000 & 5282 & 1508 & 1806 & 2200 & 1838 & 4791 & 4367 & 4942 & 4700 \\
\hline IR 87751-20-4-4-2 & 5312 & 6220 & 4250 & 5261 & 3576 & 1736 & 2333 & 2548 & 4990 & 4211 & 4940 & 4714 \\
\hline IR 87754-42-1-3-3 & 5276 & 5562 & 5000 & 5279 & 2876 & 1806 & 4000 & 2894 & 5194 & 4015 & 4915 & 4708 \\
\hline IR 87749-10-1-1-4 & 4018 & 6084 & 4900 & 5001 & 3862 & 1806 & 3900 & 3189 & 5159 & 4455 & 5570 & 5061 \\
\hline Sarju-52 & 6816 & 3823 & 5500 & 5380 & 3437 & 2153 & 4500 & 3363 & 4913 & 4110 & 5241 & 4755 \\
\hline IR 83376-B-B-71-1 & 5922 & 5119 & 4750 & 5264 & 2083 & 4167 & 3750 & 3333 & 4895 & 3914 & 5112 & 4640 \\
\hline IR 80461-B-79-3 & 5359 & 3726 & 4500 & 4528 & 4052 & 1527 & 3500 & 3026 & 4797 & 3880 & 4645 & 4441 \\
\hline IR 95781-15-1-1-4 & 4825 & 6200 & 4750 & 5258 & 4322 & 1528 & 3750 & 3200 & 5163 & 4144 & 5380 & 4896 \\
\hline IR 78875-207-B-3-B & 4421 & 6750 & 5000 & 5390 & 3767 & 2500 & 4000 & 3422 & 4400 & 4415 & 4787 & 4534 \\
\hline IR 95840-33-3-2-1 & 4760 & 5338 & 5400 & 5166 & 5516 & 3835 & 4400 & 4584 & 5256 & 4645 & 6531 & 5477 \\
\hline IR 89889-18-1-2-1 & 5333 & 6111 & 4700 & 5381 & 3297 & 1944 & 3700 & 2980 & 4892 & 4482 & 5460 & 4945 \\
\hline IR 93809-2-1-3-2 & 5375 & 4942 & 4750 & 5022 & 3574 & 1944 & 3750 & 3089 & 5254 & 4279 & 5506 & 5013 \\
\hline IR 95814-29-1-1-3 & 4302 & 5245 & 5500 & 5016 & 5092 & 2223 & 4500 & 3938 & 4820 & 4239 & 5277 & 4779 \\
\hline Sukhkha Dhan-2 & 3455 & 5022 & 4250 & 4242 & 3707 & 2777 & 3250 & 3245 & 5444 & 3954 & 5395 & 4931 \\
\hline Mean & 5175 & 5414 & 4771 & 5120 & 3665 & 2345 & 3286 & 3099 & 4945 & 4193 & 5090 & 4743 \\
\hline F-test & NS & NS & NS & $* *$ & $* *$ & $* *$ & NS & NS & NS & $*$ & NS & NS \\
\hline LSD & & & & 865.32 & 1229 & 1347 & & & & 1754 & & \\
\hline $\mathrm{CV}$ & 19.41 & 17.6 & 15.4 & 21.3 & 14.12 & 24.97 & 15.21 & 12.10 & 13 & 14.5 & 15.6 & 19.20 \\
\hline
\end{tabular}

Table 6: Straw yield (kg/ha) of drought tolerant rice genotypes over the location in different moisture condition during 2015 to 2017.

\section{Conclusion}

Higher grain yield reduction was found in reproductive stage drought (58.9\%) than normal irrigated condition. Likewise, grain yield reduction $7.9 \%$ at Surkhet and 22.7 at Doti in rainfed condition. Genotypes IR 95814-29-1-1-3, IR 95840-33-3-2-1, IR 80461B-79-3, Sukhkha Dhan-2, IR 83383-B-B-141-1 and IR 87761-391-1-4 had found higher grain yield reduction in rainfed condition than normal irrigated condition. However, genotypes IR 83376B-B-7-1, IR 877761-52-1-2-2, IR 95840-33-3-2-1 and IR 82608B-B-33-2 were found drought resistance, early maturing. These genotypes were found early maturing, high yielding and drought tolerant. So, these genotypes would help to food security in mid and far western region of Nepal. 


\section{Bibliography}

1. Lafitte H and J Bennet. "Requirement for aerobic rice. Physiological and molecular considerations". In: Bouman, B.A.M., H. Hengsdijk and B. Hardy, (Eds.) Water - Wise Rice Production. IRRI, Los Baños, Philippines (2003).

2. Kato Y., et al. "Enhancing grain yield of rice (Oryza sativa L.) under upland conditions in Japan". 4th International Crop Science Congress, Brisbane, Australia (2004).

3. Forbes JC and RD Watson. "Plants in Agriculture". Cambridge, Britain (1994): 72-78.

4. Lanceras JC., et al. "Quantitative trait loci associated with drought tolerance at reproductive stage in rice". Plant Physiology 135 (2004): 384-399.

5. Isendahl N and G Schmidt. "Drought in the MediterraneanWWF policy proposals". World Wide Fund for Nature, Adena, Madrid (2006).

6. Hongbo S., et al. "Changes of anti-oxidative enzymes and MDA content under soil water deficits among 10 wheat (Triticum aestivumL.) genotypes at maturation stage". Colloids and Surfaces B: Biointerfaces 45 (2005): 7-13.

7. Bouman BAM., et al. "Yield and water use of irrigated tropical aerobic rice systems". Agriculture and Water Management 74 (2005) 87-105.

8. Tao H., et al. "Growth and yield formation for rice (Oryza sativa L.) in the water-saving ground cover rice production system (GCRPS)". Field Crops Research 95 (2006): 1-12.

9. MoAD (Ministry of Agriculture Development). Statistical Information of Nepalese Agriculture. Agriculture Information and Communication Centre. Government of Nepal, Ministry of Agriculture Development. Kathmandu, Nepal (2018).

10. Basnet BMS. "Environment friendly technologies for increasing rice productivity". The Journal of Agriculture and Environment 9 (2008).

11. Bhattacharya T and RK Panda. "Effect of climate change on rice yield at Kharagpur, West Bengal”. OSR Journal of Agriculture and Veterinary Science 4 (2013): 06-12.

12. Gumma MK., et al. "Temporal changes in rice-growing area and their impact on livelihood over a decade; A case study of Nepal". Agriculture, Ecosystems and Environment 142 (2011): 382-392.
13. Farooq M., et al. "Advances in drought resistance of rice". Critical Reviews in Plant Sciences 28 (2009): 199-217.

14. Blum A. "Drought tolerance - Is it a complex trait?". In: Saxena NP and O'Toole JC eds. Field Screening for Drought Tolerance in Crop Plants with Emphasis on Rice: Proceedings of an International Workshop on Field Screening for Drought Tolerance in Rice (2002): 17-22.

15. Nonami H. "Plant water relations and control of cell elongation at low water potentials". Journal of Plant Research 111 (1998): 373-382.

16. Rahman MT., et al. "Effect of water stress at different growth stages on yield and yield contributing characters of transplanted Aman rice". Pakistan Journal of Biological Sciences 5 (2002): 169-172.

17. Farooq M., et al. "Physiological role of exogenously applied glycine betaine in improving drought tolerance of fine grain aromatic rice (Oryza sativa L.)". Journal of Agronomy and Crop Science 194 (2008): 325-333.

18. Jaleel CA., et al. "Alterations in morphological parameters and photosynthetic pigments responses of Catharanthus roseus under soil water deficits". Colloids Surface B. Biointerfaces 62 (2008): 312-318.

19. Carlos ACC., et al. "Grain quality of upland rice cultivars in response to cropping systems in the Brazilian tropical savanna". Scientia Agricola (Piracicaba, Braz.) 65 (2008): 468-473.

\section{Volume 3 Issue 11 November 2019 (C) All rights are reserved by Santosh Raj Tripath., et al.}

